\title{
The PRIME Project: A Proposal For Fermilab To Join A NASA SMEX
}

October 8, 2001

\author{
Spokesperson \\ Stephen Kent \\ Head, Experimental Astrophysics Group \\ 630-840-8264 (skent@fnal.gov)
}

\section{Computing Division}

Experimental Astrophysics Group

Jim Annis

Rich Kron

Brian Lee

Huan Lin

John Peoples

Chris Stoughton

Douglas Tucker

Brian Yanny

Dan VandenBerk

\section{Integrated Systems Development}

Don Petravick, Head

Don Holmgren

Eric Neilsen
Particle Physics Division

Theoretical Astrophysics Group

Scott Dodelson, Head

Josh Frieman

Albert Stebbins

Technical Centers

Bill Boroski

\section{Scientific Collaboration}

Johns Hopkins University

Fermi National Accelerator Laboratory (proposed)

Goddard Space Flight Center

Max Planck Institute for Astronomy, Heidelberg

Space Telescope Science Institute

\section{Industrial Partners}

Swales Aerospace

Space Dynamics Laboratory 


\section{INTRODUCTION}

The Primordial Explorer (PRIME) is a proposed satellite designed to conduct an infrared imaging survey of $1 / 4$ of the sky, with a principal science goal of probing the universe at redshifts greater than 1. We expect to use the data from PRIME to extend our Sloan Digital Sky Survey-based studies of the distribution of mass in the Universe to the high redshift frontier.

The PI for the proposal is Wei Zheng of the Johns Hopkins University. A preproposal was submitted to NASA's small explorer program, where it was one of 31 proposals submitted. PRIME, along with six other proposals, was selected for a full Phase A study and proposal. The proposal is due at NASA in mid-December and the final selection of two proposals for the small explorer program will be made sometime in the first half of 2002. The scheduled launch date is September 2005 and the mission is planned to have a lifetime of 2-3 years.

\section{PRIME and Fermilab}

The PRIME mission plays to the strengths of the Fermilab astrophysics endeavor. It is a natural extension of the Sloan Digital Sky Survey to higher redshift via longer wavelengths, and naturally fulfills the following criteria that the two astrophysics groups established for their next project:

(i) It complements the Sloan Digital Sky Survey (SDSS),

(ii) The skills and expertise of our groups will be a major contribution to the project, and most importantly,

(iii) It will yield science of direct interest and relevance to the mission of the Laboratory.

The research goals of the Experimental Astrophysics Group (EAG) and Theoretical Astrophysics Group (TAG) are largely aligned, and the joint science case we outline here is aimed at the longterm goal of astrophysics at Fermilab. In particular we seek to understand the fundamental physics of the Universe via understanding the processes extant at the earliest times and learning the identity and properties of the dominant forms of matter and energy. We expect that, in addition to strengthening constraints on fundamental physics such as the neutrino mass and dark matter abundance, PRIME will by, exploring redshifts of order unity, probe the time when the mysterious dark energy is most relevant. Thus we will determine whether nature has chosen a small non-zero cosmological constant or perhaps another form of vacuum energy.

Three of partner institutions in PRIME, JHU, the Max Planck Institute for Astronomy in Heidelberg, and the Space Telescope Science Institute, are also partners with Fermilab on the Sloan Digital Sky Survey. Thus, participation in PRIME will allow Fermilab to build on existing relationships with those institutions. 


\section{SCIENCE WITH PRIME}

The PRIME mission proposes to survey $1 / 4$ of the sky in 4 near-infrared bandpasses. The spacecraft flies a $0.8 \mathrm{~m}$ diameter telescope that passes light through a series of dichroics, splitting the light into 4 bandpasses and onto 8 infrared imaging arrays. The orbit is low earth and (nearly) sun-synchronous, so the anti-earthward pointing telescope views the entire celestial sphere over the course of 365 days. The size of the infrared arrays and the need for high resolution sets that fraction of the sky that can be imaged; roughly $1 / 12$ the celestial sphere per year. The baseline mission is 2 years, with a practical limit of 3 .

This recitation of facts does not do justice to the power of the PRIME mission. The core idea is that redshift at cosmological distances shifts the incredibly informative spectral range between the hydrogen transitions of Ly-alpha at $121.6 \mathrm{~nm}$ and $\mathrm{H}$-alpha at $656.3 \mathrm{~nm}$ out into the infrared. The key technical fact is that in the infrared the atmosphere of the Earth is extremely bright from a series of molecular emission lines and is completely opaque over broad spectral regions due to absorption by $\mathrm{H}_{2} \mathrm{O}$. Flying above the atmosphere produces a 1000 fold reduction of the background and allows a sub-meter class telescope to be more sensitive than a ground based 10-meter telescope. The key science fact is that in astronomy higher redshift plays the role of higher energy in physics: it is the frontier at which fundamental theory is tested against experiment.

The strength of PRIME is that it tracks that core spectral range out to high redshift in a survey that covers an appreciable fraction of the sky down to cosmologically significant flux levels. With the PRIME data set we can study very early times in the Universe by looking at the very highest redshift objects, and we can study the formation of structure over time to glean information of the amounts of dark matter and dark energy in the Universe.

\section{The Earliest Times}

In many ways PRIME is a natural extension of the Sloan Digital Sky Survey to higher redshift via longer wavelengths, and a comparison of PRIME and SDSS is illuminating.

PRIME can search for the very first quasars. The reddest SDSS filter has long wavelength cutoff of 1 micron, so quasars can be found out to $z=6.5$ (when Ly-alpha passes beyond 1 micron). Since the era of peak quasar density is between $z=2$ and $z=3$, the SDSS is already probing the formation of the quasar population. In addition the SDSS has already discovered twelve of the highest redshift quasars and one of these, the quasar at a redshift of 6.28 , is the most distant quasar observed to date. All of this comes from a data sample that we expect to be $15 \%$ of the final SDSS data. There are indications from the spectra of the $z=6.28$ SDSS quasar that the intergalactic hydrogen has significant fraction of neutral hydrogen; at lower redshifts the hydrogen in the intergalactic medium is almost fully ionized. Thus at redshift 6 the SDSS may have seen the end of the epoch of reionization. This epoch began when the first stars and quasars formed; they created the ionizing photon field capable of ionizing hydrogen and helium in the space between the large clouds that eventually became the first galaxies. PRIME is capable of finding quasars to $z=15$ or higher, the very first quasars in the Universe, and thus see the beginning of the epoch of reionization and probe its entire span. Confirmation of the candidates found in PRIME will require spectroscopy on the largest telescopes on the ground and in space and in particular it will require the Next Generation 
Space Telescope. Part of NASA's interest in PRIME derives from science goals PRIME shares with NGST; the PRIME wide field imaging survey is the natural precursor mission to the NGST deep imaging and spectroscopy mission. (Chris Stoughton, Dan Vanden Berk, EAG)

PRIME can follow the galactic population to redshifts of order 3; these are high enough redshifts that the formation models are quite uncertain. The peak era of star formation in the universe seems to be $\mathrm{z}=2$, though that is controversial because the current samples of galaxies at that redshift are mostly optically selected and therefore view the rest-frame UV; starlight at the wavelength is dominated by the presence of a few young, massive stars and can be easily extinguished by intervening dust. Infrared data allow selection from the presence of large numbers of older, less massive stars, which at low redshift make up the bulk of the stellar mass. Furthermore, longer wavelength data is less sensitive to obscuration to dust in star forming galaxies and sub-millimeter observations suggest that a large fraction of star formation is missed in optically selected surveys. Thus the infrared-selected, high-redshift galaxy sample provided by PRIME serves as a natural complement to ground-based, optically selected galaxy surveys such as SDSS. Our galaxy evolution program becomes one of tracing and understanding the star formation history of the Universe, especially in relation to the entities that become the normal galaxies in the local Universe as observed by SDSS. The PRIME filter set allows photometric redshifts to be estimated out to $\mathrm{z}=2$ for galaxies of average intrinsic luminosity, once a few thousand redshifts have been obtained for a training set. (Huan Lin, EAG)

\section{The Evolution of Structured Mass}

PRIME can make direct measurements of the large-scale structure of our galaxy. Because infrared light is exponentially less sensitive to dust absorption than optical light, PRIME can view halo stars right through the Galactic plane, and its multiple bandpasses allow precision selection of the type of star. This allows us to finish the map of the halo started with SDSS, completing the census of the large tidal remnants we have discovered there and giving us the integrated history of mass accretion into the Galaxy (Brian Yanny, EAG).

PRIME can make direct measurements of the large-scale structure of the Universe using the unique IR dataset and photometric redshifts derived from that dataset. The galaxy angular correlation function may be directly inverted to provide a measure of the matter power spectrum. The SDSS measurements of the galaxy angular correlation function have been divided into bins of median redshifts $z=0.19,0.25,0.33,0.40$. The PRIME measurements will have similar statistical power and scope, but at a factor of 2 higher $\mathrm{z}$, allowing direct measurements of the evolution of clustering and of the power spectrum (Scott Dodelson, TAG).

PRIME will allow construction of cluster and group catalogs, which map the Universe into low and high-density regions. The statistics of the clusters is another direct measure of the mass evolution, as they are the largest bound structures in the Universe, and provide the tools to examine how the mass concentrations affect the formation of galaxies. With the SDSS we can trace how local density effects galaxy evolution in situ out to $z=0.5$; the PRIME data sample will allow us to take the study out to $\mathrm{z}=2$ with very high statistical power (James Annis, Steve Kent, Brian Lee, Douglas Tucker, EAG). 
The PRIME imaging depth is extreme, and makes possible several weak lensing studies. One can stack similar objects together in order to increase the signal to noise of the weak lensing signal, a program pursued with great success in the SDSS data on individual galaxies, with outstanding promise for groups and clusters of galaxies as well. The PRIME data will allow similar stackings but at redshifts a factor of 2 higher; we will make measurements of the change of the mass profile of the median galaxy over the last 4 billion years, surely a fundamental measurement. (Albert Stebbins, TAG).

PRIME takes that extreme depth over a major fraction of the sky, making possible statistical approaches to mass evolution impossible at present. A program showing great promise is measuring the mass of individual clusters using weak lensing. This measurement depends most sensitively on the surface density of galaxies; the PRIME wide survey is comparable to the relatively small area SDSS Southern Survey, over an area a factor of 30 larger. PRIME will detect the distortion of background galaxies by the lensing of the cluster mass for modest clusters like Virgo at $0.2<=z<=0.75$, and for great clusters like Coma out to $z=1.5$. The PRIME dataset of cluster masses will be vast, allowing us to track the evolution of bound structure growth and place strong constraints on the cosmological models used to model that growth, including the amount of dark matter and the density of the dark energy (Josh Frieman, TAG, James Annis, EAG).

Finally, PRIME will allow the measurement of the purely statistical weak lensing signal of largescale structure. One averages the weak lensing signals in cells on the sky and measures the statistical power of the large-scale structure induced distortion. This measurement goes roughly as the area surveyed times the root of the galaxy surface density; this product is roughly equal for the larger SDSS Northern Survey and the deeper PRIME wide survey. Thus PRIME will make a measurement of the mass power spectrum comparable to SDSS, and provide a valuable confirmation. But interestingly enough, the large scale structure producing the lensing will have a median redshift of around $z=0.5$, to be compared with the $z=0.2$ of the SDSS measurement. This opens the window of the direct observation of the evolution of the mass power spectrum with time, the details of which also depend on dark matter and dark energy (Scott Dodelson, TAG).

There is a wide range of projects that can be done with this survey. The ones outlined above are the ones Fermilab scientists are actively working on. The names of the scientists listed after the description of each topic would be much longer if this were a list of the people interested in working on each topic.

\section{FERMILAB PARTICIPATION IN PRIME}

We propose that Fermilab become a partner in the PRIME project. Fermilab brings to this project its experience in the SDSS, including

(i) Our knowledge of the collaborative construction of astronomical science pipelines with university groups.

(ii) The experience of our data processing team with terabyte scale astronomical data.

(iii) Our knowledge of the calibration of large astronomical surveys.

(iv) Our experience with the program management of large astronomical software projects.

(v) Our experience distributing large data sets to a collaboration for analysis. 
We propose that Fermilab accept overall responsibility for data processing and contributing responsibility for some elements of mission planning.

Fermilab will be the site of the actual data processing operations. JHU and Fermilab will jointly develop the data processing pipelines. Fermilab will deliver the processed data to the Space Telescope Science Institute (STSci) for distribution to the public. The STSci will be responsible for archiving and distributing the processed data to the public. All three institutions will play almost the same roles that they play today in the Sloan Digital Sky Survey.

The PRIME satellite will produce about 5 terabytes of raw data per year for 2-3 years. This volume is comparable to that of the SDSS. However, the data are less complex than the SDSS due to the fact that there is only one (instead of four) types of detectors.

The scope of work to be done by Fermilab covers the following areas:

1. Data Processing Infrastructure: Create and maintain code infrastructure, code repository, coding standards, common software tools, code versioning and distribution framework. Define procedures for code reviews and provide training for new hires. Upgrade common pipeline code based on the existing SDSS code framework. Provide system administration for Fermilab-based development and production computing machines.

2. Science software: Prepare functional specifications for all software pipelines. Upgrade common science code based on existing SDSS code framework. Create and maintain code for instrumental calibrations derived from in-flight data, astrometric, and photometric calibrations. Design and construct a database to store object catalogs in uncalibrated form with the ability to apply calibrations as they are derived. JHU will have responsibility for development of the data processing pipeline code itself.

3. Test data/simulations: these will be the responsibility of JHU.

4. Data processing production system: Specify, procure, and manage all computing hardware, create data processing scripts and infrastructure, define software acceptance procedures, integrate the data processing pipelines with processing scripts, and perform regression tests using simulated data.

5. Mission planning: Define survey footprint and mapping strategy and develop code to provide spacecraft pointing positions vs. time based on feedback from actual orbital parameters.

6. Pipeline operations: Administer data processing production systems, run data processing jobs, create and track calibration files, run jobs to operate the database, construct a testbed for regression testing, distribute data to the collaboration and to STScI for archiving. In collaboration with JHU and TAG, design and carry out quality control and quality analysis tests to ensure data integrity. In conjunction with JHU, maintain and upgrade data processing pipelines as needed.

7. With the whole collaboration, produce compelling science with the data. Both the TAG and the EAG will lead the effort to extract science from the raw data. The ability of the two groups to work 
together and lead such efforts has recently been demonstrated with a large group of papers on the implications of the Early Data Release of SDSS for large scale structure. Six papers were released simultaneously assessing the cosmological implication of this data set. Fermilab scientists from the two groups led this effort. Among other indications of this leadership is the fact that three of these six papers had Fermilab first authors. Dozens of other papers (some forthcoming) on the early data further illustrate our ability to perform the world class analysis this caliber of data deserves.

\section{LABORATORY RESOURCES FOR PRIME}

The funding would be a combination of in-kind contribution from Fermilab (approximately $\$ 2.3$ million M\&S) and direct funding by the PRIME project (approximately $\$ 3.1$ million). The major cost to the Laboratory will be the personnel cost of the existing groups currently engaged in the SDSS. We propose that the level of effort that the Computing Division and Particle Physics Division allocate to SDSS and PRIME remain constant through 2009. As the Laboratory level of effort required to fulfill the commitment to SDSS declines, beginning in 2003, the Laboratory level of effort in PRIME will begin to grow and reach a maximum in 2007, when the SDSS Archive will have been completed. Since the Fermilab responsibilities on PRIME will require three additional FTE's between 2004 and 2008, the PRIME proposal to NASA provides for three NASA funded postdocs at Fermilab.

The principal responsibility of Fermilab to the SDSS is to process all data and distribute them to the collaboration and the Space Telescope Science Institute. EAG and TAG carry this responsibility, as well as being deeply engaged in the science of the SDSS. The combined effort from the EAG and TAG is 17 people working either part or fulltime. Expressed in FTE, about 9 FTEs are directly involved in data processing and related SDSS activities and they would eventually transition to PRIME. The Integrated Systems Development Group built and currently maintains the data acquisition system at APO. They and other groups in the Computing Division provide about 2.5 FTE in effort for data acquisition and system support. We request that this support continue at the same level of effort. The Technical Centers Department of the Particle Division provides five people. Three of these people are stationed at APO and maintain the SDSS 2.5-meter telescope. Once the SDSS is completed they could be reassigned to other projects in the Laboratory. The other two are the SDSS Project Manager and an Administrative Assistant. We request the same level of support be applied to the project management of Fermilab's responsibilities on Prime.

The SDSS is currently scheduled to terminate data collection in June 2005, with a final data release in 2006. Thus, PRIME will begin data collection shortly after the SDSS ends. The PRIME staffing plan presumes that SDSS staffing can begin ramping down in 2003 . This rampdown presumes that ongoing pipeline development end by mid 2002 and the database development ends in mid 2003. After July 2003 the SDSS will enter a purely operational mode. 


\section{CONCLUSION}

PRIME represents a major scientific opportunity to purse to high redshift the programs we began with the SDSS. Building on the lessons and success of the SDSS, we propose to perform the natural follow-on experiment, a deep infrared survey of a large fraction of the sky. For a price equal to the ongoing SDSS production, we can extend our grasp from the $z=0.4$ and $z=6$ of the SDSS to the $z=3$ and $z=15$ of PRIME. Finally, the PRIME program will span a 6 year timespan; compared with the SDSS 15 year span PRIME is quick. Its beauty lies in standing on the shoulders of the SDSS. 